\title{
Análisis feminista de las propuestas poshumanas de la tecnología patriarcal
}

Feminist analysis of the poshuman proposals of patriarchal technology Análise feminista de propostas pós-humanas da tecnologia patriarcal

Isabel TAJAHUERCE ÁNGEL

Universidad Complutense de Madrid, España / isabeltj@ccinf.ucm.es

Cristina MATEOS CASADO

Seminario de Investigación BIOTECgender,UCM, España / biotecgender@ucm.es

Rut MELERO SUSO

Seminario de Investigación BIOTECgender, UCM, España

rut@volandovengo.com

Chasqui. Revista Latinoamericana de Comunicación

N.o 135, agosto - noviembre 2017 (Sección Monográfico, pp. 123-141)

ISSN 1390-1079 / e-ISSN 1390-924X

Ecuador: CIESPAL

Recibido: 22-05-2017 / Aprobado: 31-08-2017 


\title{
Resumen
}

Este trabajo indaga en las propuestas poshumanas de la tecnología desde una perspectiva de género, en el marco de la teoría feminista de las Ciencias Sociales. Examina la nueva "articulación" de la vida a través de la tecnología, realizando un acercamiento crítico al androcentrismo en el tratamiento que los medios periodísticos y el género de ciencia ficción aplican a las cuestiones relacionadas con los avances tecnológicos y la transformación humana, desde la robótica y lo ciborg. El artículo finaliza con un análisis de la serie sueca de ciencia ficción Real Humans (2012-2014), en donde robots denominados hubots (androides) conviven a diario y se relacionan con personas humanas.

Palabras clave: roles de género; robótica; inteligencia artificial; ciencia ficción, androcentrismo.

\begin{abstract}
This work looks into the posthuman proposals of technology from a gender perspective and within the feminist framework of social sciences. It analyses the regulation of life by technology from a critical standpoint on the treatment that media and the science fiction give to androcentricity when addressing technological progress and human transformation, from robotics to cyborg. The article concludes analyzing the Swedish science fiction series called Real Humans (2012-2104) in which robots, called hubots (androids), share their daily life and get along with humans.
\end{abstract}

Keywords: gender roles; robotics; artificial intelligence; science fiction; androcentricity.

\section{Resumo}

Este trabalho indaga a respeito de propostas pós-humanas da tecnologia desde uma perspectiva de gênero, no âmbito da teoria feminista das ciências sociais. Examina a nova "articulação" da vida por meio da tecnologia e propõe uma aproximação crítica ao androcentrismo presente no tratamento que os meios de comunicação e de ficção científica aplicam às questões relacionadas com os avanços tecnológicos e a transformação humana, a partir da robótica e o ciborgue. $\mathrm{O}$ artigo finaliza com uma análise da série sueca de ficção científica Real Humans (2012-2014), onde robôs denominados hubots (androides) convivem diariamente e se relacionam com humanos.

Palavras-chave: papéis de gênero, robótica, inteligência artificial, ficção científica, Androcentrismo. 


\section{Introducción: la robótica, lo ciborg y ciber como objetos de estudio en la investigación feminista}

El feminismo ha venido cuestionando sistemáticamente aspectos generales de la ciencia y el desarrollo tecnológico, como el monopolio de los hombres en la producción del conocimiento científico, los cánones de "alta ciencia”, la idea de "progreso" masculino, o cómo se puede hacer ciencia sin que exista en la comunidad científica una democracia interna que reconozca a las mujeres como sujetos de conocimiento (Pérez \& García, 2017). Y en este sentido, planteándose preguntas inquietantes para el futuro de las mujeres bajo el signo de la ciencia y tecnología: ¿A qué tipo de progreso se orienta la ciencia y tecnología? ¿A quién/ quiénes benefician los avances científicos y tecnológicos? ¿Es posible fracturar el simbolismo originario o genérico de una ciencia basada en una ideología masculina, binaria, violenta y misógina? ¿Es posible pensar en el "progreso" y en el "desarrollo" desvinculados de las tecnologías de la reproducción que agreden los cuerpos y las vidas presentes y futuras de las mujeres? Para algunas feministas como Germaine Greer (1970) “los avances médicos desde la anticoncepción a la genética dejan a las mujeres inermes, en manos de la química, la mutilación, y lo que llama el establishment patriarcal de la reproducción" (citado en Rivière, 2000). Judy Wajcman afirma que las "modificaciones" del ser humano no llevan implícitas la transgresión del orden genérico asignado porque en muchos casos afirma "se utilizan para reforzar los estereotipos de género más que para subvertirlos" (Wajcman, 2006, p. 141). Feministas poscoloniales como Audre Lorde, se posicionaban tajantemente hace más treinta años respecto a la tecnología, refiriéndose a que las prótesis de mama tras un cáncer eliminaban la política de la diferencia, ya que "la prótesis ofrece el consuelo vacío de "nadie va a notar la diferencia'. Pero es precisamente esa diferencia la que yo quiero afirmar, porque la he vivido, y sobrevivido" (Lorde, 1980, p. 52).

Desde el protofeminismo de Mary Shelly, con su obra Frankenstein (1818) el feminismo ha ido incorporando en sus diferentes corrientes el impacto de la tecnología en la vida de las mujeres. La palabra ciborg es la unión de las palabras cybernetics y organism. El neologismo fue desarrollado por Manfred Clynes y Nathan Kline, este último pionero en la psicofarmacología, especialmente la referida a los tratamientos antidepresivos ${ }^{1}$. El concepto ciborg se desarrolló en Astronautics (1960) durante la carrera espacial (1957-1975) ${ }^{2}$. El concepto fue estudiado durante una década hasta que se abandonó, vinculándolo con el espa-

1 Deberíamos plantearnos profundizar en las vinculaciones históricas ya que el "padre" del "ciborg patriarcal" es el mismo "padre" de los ansiolíticos, uno de los fármacos más administrados a las mujeres, como indica Carme Valls Llobet, autora del libro Mujeres, salud y poder (2009), en donde aborda entre otras cuestiones la creciente medicalización de los cuerpos de las mujeres.

2 Es importante señalar en la Carrera Espacial norteamericana y en la NASA la presencia de mujeres, especialmente de mujeres afroamericanas, tal y como recoge la película Figuras ocultas (2016), basada en la recuperación del trabajo pionero de la matemática afroamericana Katherine Johnson, y sus compañeras, la matemática, Dorothy Vaughan y la matemática e ingeniera aeroespacial Mary Jackson. 
cio para referirse al híbrido "hombre-máquina" de los trajes espaciales de los astronautas a los que la prensa del momento se refería como robot man.

Después de esa primera vinculación del concepto ciborg a la ciencia patriarcal, en los años '8o fue recuperado por Donna Haraway (1983) y más tarde, por la ciencia-ficción y el cómic. En 1983 la autora publica A Cyborg Manifesto: Science, Technology, and Socialist-Feminism in the Late Twentieth Century, obra fundamental que rompería con las formas clásicas de concebir las determinaciones sexo genéricas, e introduciría el concepto de ciborg y, por tanto, la condición híbrida.

Esta nueva visión del mundo generaría, según autoras como Celia Amorós, una "nueva forma de ciudadanía", nuevas relaciones basadas en la idea de "articulación" y no de identidad. En estas nuevas formas de "articulación" afirma que las empresas de biotecnología decidirán "qué mundos habitables se construirán, para quién, y quién vivirá y quién morirá” (Amorós, 2007, p. 20). Una nueva política de la vida y del futuro en donde las nuevas "articulaciones" sustituirán a las antiguas bajo una nueva ética. Rosi Braidotti (1994) y otras autoras han definido una nueva ética a la que denominan "ética nómada", llena de múltiples cosmovisiones y transposiciones (Braidotti, 2006) que daría origen a un nuevo sujeto al que denominan "posthumano" (Braidotti, 2013). Este "posthumano político" propondría nuevas dimensiones sociales y políticas frente a la pervivencia del "binarismo social y sexual" 3 como un mecanismo de poder del orden social patriarcal, o como un "dispositivo de poder" (Foucault, 1975).

En relación a la computación fueron fundamentales las aportaciones de Ada Lovelace (1815-1852) 4 , sería la pionera de la computación al describir por primera vez el lenguaje de la programación y dejar constancia científica del impacto que podría suponer para la sociedad.

Aunque lo ciber se puso de moda en los años 80, la ciencia cibernética se venía desarrollando desde finales de los 40, después de la publicación de la obra de Norbert Wiener (1948) Cibernética o el control y comunicación en animales y máquinas. Dentro del feminismo éste ha sido el concepto más desarrollado en la teoría y en la práctica, generando nuevos términos como ciberacoso o ciberviolencia. De todas las áreas tecnológicas, el ciberespacio es la que mejor visibiliza la lucha sexo-genérica, y denuncia el hostigamiento, al mismo tiempo que reproduce las violencias contra las mujeres tal como expresa Diana Fernández Romero:

3 Entendemos "binarismo" como un pensamiento hegemónico que representa la realidad desde una concepción dicotómica y opositiva, basada en la idea genérica de creación del mundo y de complementariedad de los sexos, que ha consolidado una jerarquía sexual. Una superestructura del poder, lo que Monique Wittig denominó como un "pensamiento de la dominación" (Wittig, 1992, p. 54). Para saber más sobre el concepto binarismo se recomienda la lectura del glosario Barbarismos Queer (Ortega, Rosón \& Platero, 2017).

4 Vinculada a su vida se estrenó la película Conceiving Ada (1997), de la directora de ciencia ficción feminista Lynn Hershman- Leeson http://www.lynnhershman.com/. 
[...] muchas activistas han tomado el ciberespacio como un lugar desde el que interpelar y subvertir el discurso en la red [...]. Muchos troles reaccionarios lo invaden constantemente hostigando y acusando de feminazis o de hembristas a las mujeres que utilizan la red para hacer activismo feminista. (Fernández Romero, 2016)

Viginia Woolf autora de "Una habitación propia" (1929) afirmaba que "una mujer debe tener dinero y una habitación propia si desea escribir ficción". Los mundos "abiertos" y aparentemente accesibles del ciberespacio no han llegado aún a todas las mujeres, pero sí han permitido a muchas el primer acceso a "una habitación propia”, desde donde mirar mundos restringidos en sus sociedades. Para otras, ha generado una multiplicidad de "habitaciones propias", de espacios que habitar, pensar, o para generar redes de acción. Lo ciber se ha convertido en un espacio que libera, que denuncia la opresión de las mujeres en el mundo y visibiliza su situación y, al mismo tiempo, se han convertido en un espacio minado que amenaza sus vidas, como en el caso de la empresaria digital Roya Mahboob, perseguida por el integrismo y bajo amenaza de muerte por ser una referente árabe de la empresa digital, y haber sido elegida en el año 2013 como una de las mujeres más influyentes del planeta por la revista Time. Mahboob fundó Digital Citizen Fund, un proyecto destinado a la alfabetización digital de adolescentes afganas, y a la expresión y proyección de las mujeres a través de las plataformas tecnológicas (Ruiz del Árbol, 2016).

En relación a la inteligencia artificial y la robótica no existen investigaciones feministas ni autoras que hayan incorporado al sector la perspectiva de género. La palabra robot apareció por primera vez en la obra Robots Universales Rossum R.U.R. (1920) del escritor Karel Čapek. La palabra "robot" derivaría según la filología del antiguo eslavo de "esclavo", y la palabra "robota" del checo que significa "trabajo". Ambos significados, con clara referencia a la servidumbre, como más adelante se podrá ver representado en el análisis de la serie de televisión Real Humans.

\section{Una aproximación a los medios de comunicación y el impacto de los avances científicos y tecnológicos relacionados con la transformación de lo "humano"}

La ciencia y los avances tecnológicos siempre han ocupado un espacio significativo en los medios periodísticos y otros medios de comunicación social. Contar los avances tecnológicos implica tener una adecuada formación para transmitir de forma veraz el impacto y las consecuencias que pueden tener en la sociedad, fomentando un debate crítico y un análisis ético. Desde el siglo XIX la información no especializada sobre ciencia y tecnología ha caído en el sensacionalismo, incrementando la brecha entre ciudadanía y ciencia, sin profundizar en la re- 
percusión social de los avances más innovadores, ni incorporar un pensamiento crítico y riguroso, y muchos menos, un análisis de género.

A las mujeres se les sigue reservando espacios "imaginarios" vinculados con lo doméstico. Aunque "nadie pretende o busca sexualidad a una lavadora" (Escudero Pérez, 2010, p. 51), la lavadora tiene una asignación social del género, al igual que un taladro. El género y sus asignaciones en el ámbito público y privado se mantienen hoy, por lo que introducir la perspectiva de género en la ciencia y la tecnología se presenta como una tarea prioritaria.

En las noticias recientes sobre tecnología en medios digitales de diversos países, se observan sesgos de género y reproducción de un sistema androcéntrico y desigual. En algunos medios españoles de amplia difusión sorprenden las imágenes sexualizadas que acompañan a la noticia. Es especialmente significativa la fotografía que se incluye en el artículo titulado "Retos de un futuro posthumano" (Cortina \& Serra, 2016) con una imagen sexualizada de la atleta y modelo Aimee Mullins, muy distinta de la publicada por el mismo periódico mostrando al científico Hugh Herr con sus piernas biónicas (Snyder, 2016).

Figura 1. De la izquierda a derecha, imagen de la atleta y modelo Aimee Mullins y del científico Hugh He rr.
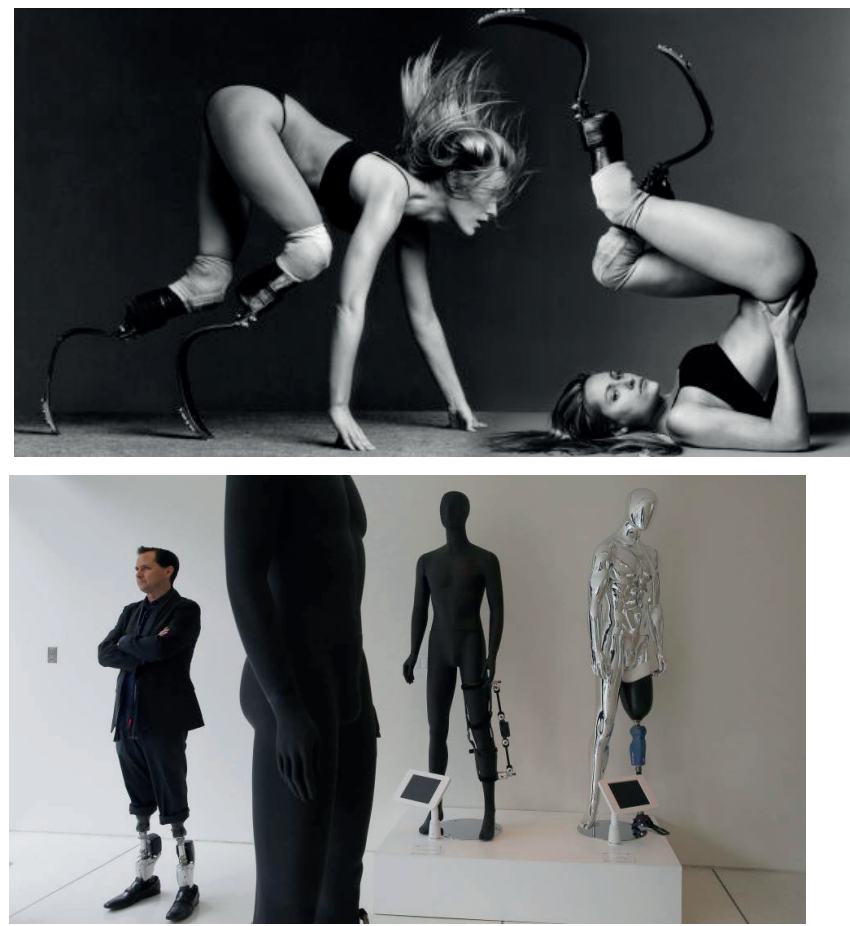

Imagen: El País. 
La revista Time incluye un reportaje sobre la modelo biónica Rebekah Marine, introduciendo exclusivamente el "lado humano" (Most, 2015). El "lado humano" en los implantes biónicos es habitual en la prensa, así lo recoge The New York Times, en "Hand of a Superhero" (Mrozfeb, 2015), y otros periódicos de América Latina consultados como El Comercio de Ecuador ("Las prótesis le dan una nueva vida a Jessica”, 2014). La reflexión sobre los avances tecnológicos para la vida de las personas es prioritaria en la mayoría de los medios, incluso en los periódicos con titulares más sensacionalistas y en los que los hombres aparecen con más poder, como el caso de Hugh Herr o los ingenieros a los que se muestra o cita. Las mujeres son casi invisibles, salvo en las historias humanas, y la mayoría con implantes biónicos parecen ser bailarinas o modelos.

El lenguaje también transmite imaginarios sutiles que construyen un mundo de poder o de sometimiento. En titulares como "De posthumanos y de hombres" (C. Fanjul, 2015) o "En 2045 el hombre será inmortal” (Gómez, 2014), se visibiliza al hombre y se ignora a las mujeres al nombrar en masculino, ocultando al 50\% de la población en el mundo del futuro. Sorprende la utilización de "hombre" referido al futuro tecnológico y no de "humanidad". Lo mismo ocurre si leemos algunos titulares de periódicos de distintos países de América Latina, que presentan a los robots como "aliados del hombre" (EFE, 2017) o como el "binomio hombre-máquina" (EFE, 2016). Destacan también los titulares sensacionalistas sobre la posibilidad de que los robots se rebelen contra el hombre, refiriéndose a la necesidad de una reglamentación europea sobre robots ““ ¿Y sí los robots se rebelan contra el hombre? Europa quiere un botón para 'apagarlos' en caso de peligro", 2017). El lenguaje y la ciencia siguen en masculino, mientras la tecnología evoluciona hacia lo que hasta hace poco era ciencia-ficción sin mostrar signos de cambio sexo-genérico, con estereotipos y prejuicios propios de siglos anteriores y previos a la legislación nacional, europea o internacional en materia de igualdad. La ciencia sigue siendo un concepto masculino en los imaginarios sociales, no porque las mujeres no investiguen, sino porque no son reconocidas socialmente. Las científicas reconocidas en premios internacionales son una excepción siempre destacable, como señala el diario español El País, acompañando la noticia con una imagen llena de simbolismo, de la reunión de Premios Nobel en Alemania, con el titular "Los hombres han ganado el 97\% de los Nobel de ciencia desde 1901” (Ansede, 2016). 
Figura 2. Reunión de premios Nobel en Lindau (Suiza) en 2015. La mujer que aparece no es científica, es una condesa según informa el diario.

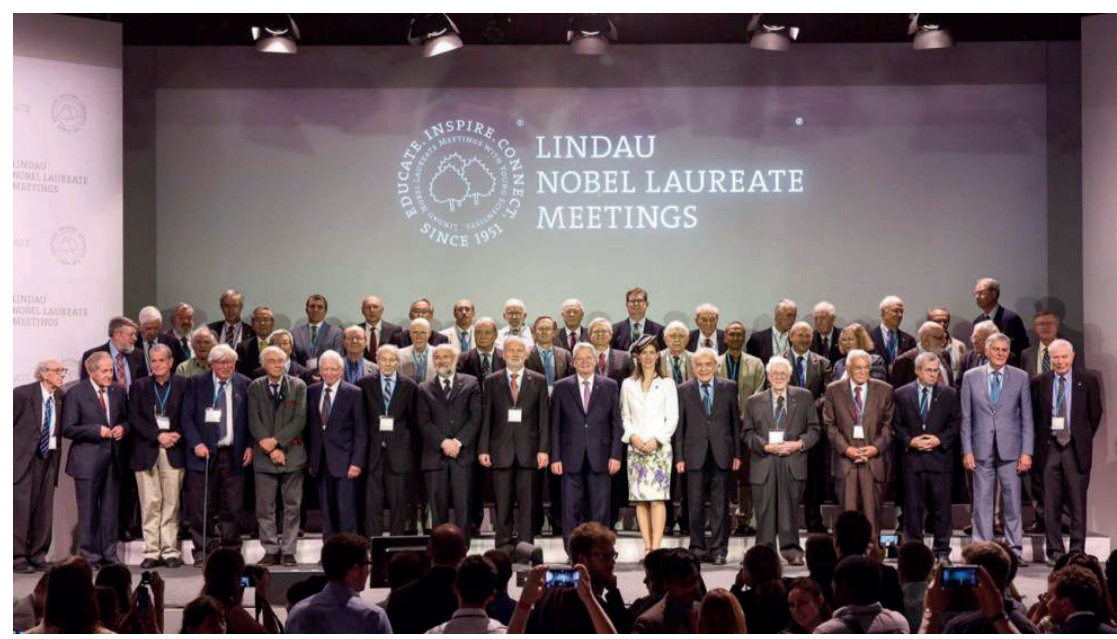

Imagen: El País.

En La Nación de Costa Rica, se profundiza en la necesidad de incorporar a mujeres a la tecnología basándose en datos que avalan que "las niñas comienzan a alejarse de los cursos de Science, Technology, Engineering \& Mathematics, STEM en la primaria porque la ciencia y la tecnología suelen considerarse áreas de dominio masculino ("Por más mujeres en tecnología", 2016).

El sensacionalismo es una constante en la prensa española y en periódicos de otros países. Son muchos los periódicos que introducen artículos sobre "sexo con robots" describiendo ya una patología específica "Robofilia: la perspectiva del sexo con robots abre dilemas morales" (Rius, 2016). El tema da para muchas páginas en periódicos del mundo que se plantean una misma pregunta “¿Podrías llegar a amar a un robot?" Este titular con la misma imagen se ha reproducido en periódicos de diferentes culturas como BBC Mundo o La Nación de Argentina, y en muchos noticiarios. Incluso periódicos y semanarios deportivos dedican espacio al tema de "amor y sexo" con robot, asegurando que en 2017 se comercializarán ya robots sexuales con apariencia humana y a mitad de siglo podrían casarse ("El sexo con robots está más cerca de lo que crees, 2016). El autor parece desconocer que ya se comercializan los "robots sexuales" que se han convertido en una potente industria (Tajahuerce \& Mateos, 2016). Recientemente en Barcelona se abrió el primer prostíbulo de Europa de muñecas sexuales hiperrealistas, con el nombre de "Lumidolls", que fue ampliamente recogido en prensa "Así pasé una hora con una muñeca por 80 euros en el prostíbulo de las sex dolls" (López Frías, 2017). La sexualidad heteropatriarcal es siempre un tema recurrente, como lo es el amor romántico, con unos toques permanentes de sensacionalismo cuando no de amarillismo. 


\section{Aproximación a la ciencia ficción desde una perspectiva de género}

En los años 70, Sam Lundwall, promotor de obras de ciencia-ficción y fantasía, revelaba el perverso y subordinado papel que tradicionalmente se le ha asignado a la mujer con la metafórica frase "los roles sexuales en la ciencia ficción son tan inalterables como el metal del casco de la nave espacial, y la emancipación una palabra desconocida" (Lundwall, 1976). La realidad es que la primera obra de ciencia ficción Frankenstein o el moderno Prometeo (1818) fue escrita por una mujer con tan sólo 20 años, la escritora inglesa Mary Shelley. La historia oficial se encargó de invisibilizar una vez más las aportaciones de las mujeres, al considerar como "padres" de la ciencia ficción a Julio Verne o a Hugo Gernsback entre otros, sin conceder el mismo estatus de reconocimiento al trabajo de Mary Shelly, que había abordado ya medio siglo antes la creación de vida humana a través de métodos científicos.

Adentrarse en la ciencia ficción implica preguntarse por el futuro, la ciencia y la ficción. Para profundizar en la idea de futuro, desde una perspectiva de género, lo haremos de la mano de "el futurismo", un movimiento que pretendía romper con el pasado y mirar con belleza y velocidad al futuro. Uno de las primeras vanguardias en donde se hizo manifiesta "una mayor pertinencia de mujeres creadoras" a pesar de que muchas respondieron a la misoginia de su tiempo, dando a entender que en el "futurismo no hay futuro para las mujeres" (Muiña, 2016, p. 16)5. Otras como Mina Loy incorporaron en sus obras los problemas de las mujeres y la transgresión del futuro marcado para ellas. Qué es el futuro y quiénes lo proyectan, tiene una vinculación fundamental con nuestro pasado y con nuestro presente desde una mirada de género. La obra, Historia de las mujeres. Una historia propia, constató de forma crítica la mayor estrategia de invisibilización de las mujeres: "no puede haber igualdad cuando más de la mitad del género humano carece de historia” (Anderson \& Zinsser, 2009, p. 21).

La ciencia ficción nos lleva también a cuestionar la ciencia como lo hizo Thomas S. Kuhn quien la revolucionó en los años 6o, con su obra La estructura de las revoluciones científicas (1962). No se le consideraba feminista, sin embargo sus teorías ayudaron a desmitificar la visión idílica de la ciencia, poniendo de manifiesto su inherente punto de vista androcéntrico, situando el foco en la conducta y procedimiento de los científicos, evidenciando el carácter humano y por ello "partidista" e "insuficiente" de cualquier elaboración de la ciencia. La crítica abierta por las Ciencias Sociales y dentro de éstas por la Teoría Feminista sobre otras maneras de hacer ciencia, revelan cómo la perspectiva masculina ha sido la medida universal. A lo largo de la historia, las mujeres han sido invisibilizadas en las diferentes áreas del conocimiento. La ciencia, sea cual sea su área de estudio, no escapa a este fenómeno, y la construcción de la ciencia ficción,

5 En 1912 se publica "Manifiesto de la Mujer Futurista" de Valentine de Saint-Point que por primera vez se recoge su traducción al español en el libro "Mina Loy. Futurismo, dadá y surrealismo" (Muiña, 2016) 
subgénero que pretende estar a mitad de camino entre el invento y el conocimiento, tampoco.

Siguiendo con el análisis futuro/ ciencia/ ficción, reflexionamos sobre los significados y significantes de la propia ficción. Las ficciones han contribuido a normalizar modelos masculinos violentos que han ido construyendo una realidad en donde no se sabe dónde acaba la realidad y comienza la ficción, o al contrario. En esas ficciones los estereotipos no cambian de naturaleza ya que esos artefactos culturales son concebidos bajo los estereotipos de sus creadoras o creadores, y por extensión, resultado de sus violencias. La ficción engendra ficción, y esa ficción engendra realidades; y como resultado podemos encontrar, por ejemplo, hombres adolescentes consumidores activos de videojuegos basados en premiar asesinatos sistemáticos o violaciones. Un paradigma que muestra la importancia de transformar los hábitos de consumo de nuestras ficciones y de nuestro arte, y aún con más urgencia, la construcción ética de "artefactos" artísticos y culturales.

La primera persona que acuñó el término science fiction fue el escritor Hugo Gernsback con la publicación de la que sería una de las primeras y más influyentes revistas del género Amazing Stories (1926). La mayoría de la obras generadas en el apogeo de la ciencia ficción estaban escritas por hombres, y los personajes femeninos solían acompañar a los masculinos como objetos pasivos. El auge de autoras de ciencia ficción, tuvo lugar en los años 60 y 70, cuando el género comenzó a reflexionar y reinventarse con líneas de pensamiento y crítica social. El género permitió dar vida a mundos que situaban a las mujeres como activas y subversivas dentro de una cultura patriarcal y androcéntrica, como reivindicaron en sus obras Ursula K. Le Guin o Andre Norton, ambas galardonadas por la Science Fiction and Fantasy Writers of America. Otras autoras reconocidas de la ciencia ficción son las estadounidenses James Tiptree, Octavia Estelle Butler, Lois McMaster, Ann Leckie, Connie Willis, Lisa Gracia Tuttle, Joanna Russ; las españolas Lola Robles y Elia Barceló; la argentina Angélica Gorodischer; la cubana Daína Chaviano; y la canadiense Margaret Atwood, quien a pesar de ser una de las autoras más reconocidas en la actualidad y cuya obra se vincula a la ciencia ficción especulativa, ha rechazado en varias ocasiones esta etiqueta o categoría, asegurando en referencia a varios de sus libros que no habla de futuro porque todo lo que muestra ya ha pasado, ya que la ficción "toma siempre aspectos de nuestra realidad y los exagera" (Seoane, 2017). En las escritoras jóvenes de ciencia ficción destaca su versatilidad y compromiso social, un ejemplo de ello es Mónica Byrne, quien ganó en 2014, el premio James Tiptree Jr. Award, y cuya obra se caracteriza por confrontar estereotipos raciales, sociales y de género (TED, 2016).

Hay que preguntarse si quienes crean ciencia ficción, al construir mundos posibles e imaginarios, tienen responsabilidad de transcender los estereotipos, los mundos binarios y las violencias. Algunas investigaciones como la de Jimena Escudero Pérez (2010), analiza "la incidencia que tienen las construcciones de feminidad resultantes del género construido sobre la articulación de personajes 
femeninos en una selección de títulos mainstream del cine de ciencia ficción" (Escudero Pérez, 2010, p. 193) que corresponde a las películas Blade Runner, Ghost in the Shell, Terminator, y Alien, concluyendo que el cine de ciencia ficción ha sido pionero en presentar modelos alternativos de feminidad e identidad sexual. Sin embargo, consideramos que para trabajar en esa línea de afirmación científica es necesario profundizar desde un análisis sexo genérico en las películas de ciencia ficción más comerciales a lo largo de la historia, desde los años veinte como El Golem (Galeen y Boese, 1920, Alemania); Las manos de Orlac (Robert Wiene, 1924, Alemania); Metrópolis (Fritz Lang, 1927, Alemania), hasta actuales como Ex machina (Alex Garland, 2015, Reino Unido) o Ghost in the shell - Agente especial ciborg (Rupert Sanders, 2017, Estados Unidos), en donde las mujeres tienen un papel más protagonista, consideradas por algunos críticos como "ciencia ficción feminista".

Existen algunos análisis y recopilaciones fílmicas que no incorporan la perspectiva de género pero que son de gran interés, como la realizada por Santiago Koval en La condición poshumana (2008).

Las series televisivas de ciencia ficción aparecen en los años sesenta, destacando hasta nuestros días: Doctor Who (Reino Unido, 1963-1989/ 2005- actualidad); The Six Million Dollar Man (Estados Unidos, 1973-1978); Battlestar Galactica (Estados Unidos-Reino Unido-Canadá, 2004-2009); Humans (Estados Unidos, 2015), adaptación americana de la serie sueca Real Humans; Westworld (Estados Unidos, 2016), basada en la película del mismo nombre dirigida por Michael Crichton. (1973); Brave New World (2016), es una adaptación de la novela clásica de ciencia ficción Un mundo feliz de Aldous Huxley (1932); y Real Humans (Suecia, 2012), serie sobre la que hemos realizado el análisis que a continuación se presenta y que permite reflexionar sobre mundos imaginarios y reales de un futuro inmediato.

\section{Análisis de la serie Real Humans: reproducción del poder y el sometimiento como bases para la sociedad del futuro}

La serie sueca de ciencia ficción Real Humans (Äkta människor), dirigida por Lars Lundström, se estrenó en 2012 y finalizó en 2014. La serie narra la historia de una sociedad en la que se han incorporado en la vida cotidiana robots, llamados hubots que cumplen diferentes funciones vinculadas en principio al ámbito laboral._La crítica social está presente a lo largo de toda la trama, sin embargo, aunque parece incorporar la perspectiva de género y su protagonista es una mujer con valores feministas, la abogada Inger Engman, un análisis en profundidad permite descubrir la perpetuación de una estructura social androcéntrica y eurocentrista que, lejos de plantear modelos alternativos superando las relaciones de poder a través del desarrollo científico y tecnológico, reproduce desigualdad y violencia como si fuese algo intrínseco a las sociedades. 
Para el análisis de la serie, se tomarán algunos aspectos destacables que se manifiestan como una constante, y que se pondrán en relación con la Teoría Feminista y con la estructura social nórdica.

\subsection{Lo que la tecnología ha unido, lo separará el Hombre: la sociedad binaria} de hubots y personas humanas

En Real Humans, los avances tecnológicos no logran evitar a nivel social una distinción y una jerarquía discriminatoria, a pesar de que en la robótica ya no hay límites y dentro de pocos años no habrá distinciones entre humanos y robots (Guillén, 2016). La serie plantea una nueva jerarquía social y sexual entre hubots y personas humanas. Las clásicas jerarquías establecidas por la raza, la clase o la orientación sexual parecen en la serie haber sido superadas, en parte, por una nueva forma de discriminación que se impone sobre el resto y que ha sido establecida por los avances tecnológicos, enfrentando dos mundos, el de las personas humanas "de verdad" y las "máquinas con apariencia humana". De tal manera que hace manifiesta la tecnofobia en una buena parte de los personajes, y la discriminación y el odio hacia los hubots que consideran que están reemplazando a los seres humanos, para lo que un grupo radical crea el "Frente de Liberación de Auténticos Humanos" que aglutina a personas con ideas integristas y de exterminio de hubots.

Figura 3. Imagen aparecida en la primera temporada de la serie Real Humans (2012) que identifica al "Frente de Liberación de Auténticos Humanos”.

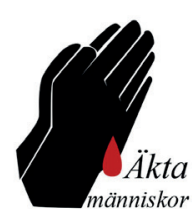

De las jerarquías clásicas, la única que se mantiene como inmutable en la serie es la sexo genérica. Tanto las hubots mujeres y las mujeres humanas, como los hubots hombres y los hombres humanos, responden a roles, prejuicios y estereotipos asignados tradicionalmente. Como se señala en el artículo Simulaciones sexo genéricas, bebés reborn y muñecas eróticas hiperrealistas, hasta el momento, "el proceso de tecnificación de las vidas y de los cuerpos no ha logrado superar las divisiones binarias, las visiones androcéntricas y la lógica tradicional patriarcal, a pesar de las innovadoras teorías de Firestone y Haraway" (Tajahuerce \& Mateos, 2016, p. 210). 
Los hubots están creados con altas capacidades para desarrollar funciones concretas, destacando especialmente las relacionadas con el trabajo y el sexo. A lo largo de las dos temporadas, especialmente en la primera, se presenta un constante enfrentamiento en el desarrollo de las relaciones personales, amorosas y sexuales, y sobre todo, un conflicto generado en el ámbito laboral. El conflicto en las relaciones laborales no sólo queda constatado en la ficción sino que ya está presente en la realidad de nuestro tiempo:

[...] la digitalización y la robotización no sólo crearán menos puestos de trabajo de los que destruyen, sino que producirán alteraciones profundas en la misma idea de trabajo, en las clases medias y trabajadoras, en las costumbres sociales y en nuestra propia visión sobre el mundo. (San Pedro, 2016)

\subsection{La religión encarnada en "los hijos de David"}

La serie representa cuatro planos en un mismo mundo; los seres humanos, los hubots (robot), los clones tecnológicos (igualmente máquinas pero copias de personas concretas en donde la clonación se produce de forma tecnológica y no por clonación celular), y los llamados "hijos de David". Esos "hijos" son un producto resultado de experimentos de inteligencia artificial desarrollados por el científico David Eischer, personaje que adquiere un permanente protagonismo en cada uno de los capítulos aunque apenas aparezca físicamente. Los "hijos a los que da la vida" son seres superiores a los hubots ya que les da la posibilidad de vivir libres (sin depender de una persona) y tener conciencia. Son creados a partir de las pérdidas de familiares del propio científico o de otras personas, y portan emociones y recuerdos. Desde una visión de género, lo primero que resulta especialmente llamativo es la reproducción de un imaginario patriarcal basado en la idea de un Dios creador "David". David representa un dios seudo religioso porque es el que da la vida a un grupo excepcional que en el cristianismo se representa como "los elegidos de Dios". "David" es considerado profeta. También reproduce a la perfección la idea androcéntrica de la ciencia fundamentada en el hombre como "creador de todas las cosas", y el poder del padre o pater familias, es decir, la propiedad del padre sobre los hijos e hijas. Al respecto, Jimena Escudero afirma en su investigación sobre ciencia ficción desde una perspectiva de género que "el robot calma nuestros anhelos divinos de creación" (Escudero Pérez, 2010, p. 46). Por tanto, como es habitual, se presenta la ciencia y la tecnología permeadas por la idea religiosa de la creación. Pero la religión, además, tiene otra presencia constante como por ejemplo, a través del personaje de la mujer pastora que vive con su pareja mujer, con una falsa apariencia de superación de roles de género y de relaciones de pareja. Por otro lado, encontramos permanentes frases y comentarios de carácter religioso como "no tienen sentimientos, ni Dios, ni educación, ni nada” (Temporada 1, capítulo 2, 34’), en referencia a la "inferioridad" de los hubots frente a los humanos por cuestiones morales. Es necesario señalar que Suecia posee libertad religiosa aunque ha destacado 
en su historia el posicionamiento de la iglesia luterana a la que pertenecen las tres cuartas partes de la sociedad. En el año 2013 la iglesia luterana sueca eligió a Antje Jackelén como primera mujer arzobispa, aunque ya en Noruega y Estados Unidos habían sido nombradas mujeres con anterioridad.

\subsection{El mito del amor romántico y los roles sexo genéricos en hubots y personas humanas}

Una parte de los "hijos de David" recuerdan la imagen física estereotipada de hombres y mujeres en la sociedad actual, reproduciendo un imaginario de "perfección" similar al de "Barbie y Ken", especialmente el personaje de Flash (Florentine) y de Gordon ${ }^{6}$. En las relaciones amorosas se generan mundos binarios, lo que se entiende por "amor verdadero" y lo que no lo es, los intereses y las relaciones sexuales o las de poder, teniendo en cuenta el origen biológico o tecnológico. El modelo de amor al que se da más protagonismo en la serie es el amor romántico, especialmente desarrollado en el personaje de Flash (Florentine) que es también quien defiende con perseverancia valores tradicionales asignados a la feminidad, especialmente de heteronormatividad, belleza y maternidad.

En el caso de dos mujeres que mantienen relaciones con hubots se cuestionan si eso es amor "verdadero" o si eso es un amor "enfermo", sin embargo, los hombres que mantienen relaciones sexuales con hubots en ningún momento se plantean cuestiones morales o éticas, porque esas relaciones son sólo sexuales, respondiendo en ambos casos a la socialización de género. Nuevamente queda reproducida la jerarquización sexual definida por Gayle Rubin (1984) y que define bajo la norma de lo que se denomina socialmente como "sexo bueno", frente a la estigmatización de lo que se denomina "sexo malo".

El amor romántico es representado en el hijo del científico David Eischer, hacia una de las "hijas de David". Aparentemente rompe con todos los estereotipos: es mayor que él, con rasgos asiáticos, y hubot. Además de portar rasgos muy marcados alejados de la construcción de la masculinidad normativa: honestidad, bondad, compromiso, ternura, y amor sin sexo.

\subsection{Simulando los roles de género}

En principio la serie parece desarrollar relaciones familiares que subvierten también los roles de género como en el caso de la familia protagonista, en donde la mujer es la que trabaja como abogada y el marido se encuentra desempleado. Sin embargo, en el desarrollo cotidiano, ella sigue realizando una doble jornada en el trabajo y en la atención y cuidados en el ámbito doméstico, mientras que su marido no llega a asumir todas las responsabilidades del ámbito de lo privado, como tradicionalmente se le asigna a una mujer que no trabaja fuera del

6 Con la elección de tan característicos nombres, queda demostrado el homenaje al cómic de ciencia ficción Flash Gordon, creado en 1934 por Alex Raymond, en donde los personajes femeninos, a pesar de su valentía y relevancia en las tramas, quedaban relegados a un segundo plano, vinculados a historias románticas con los protagonistas u objetualizados. 
hogar. Por otro lado, todas las cuestiones de carácter "emocional" de la familia recaen sobre la "madre" porque el "padre" tiene una permanente incapacidad para resolver los conflictos. Representando el llamado "velo de la igualdad" presente también en sociedades pioneras en las políticas de igualdad y corresponsabilidad.

\subsection{La transgresión de la norma de género: mujeres "malas"}

Las hubots liberadas que tienen poder se diseñan basándose en estereotipos sexistas, convirtiéndolas en malvadas, manipuladoras y perversas, como Beatrice y Niska. Las hubot más violentas serán las que mantengan relaciones con los varones humanos para lograr fines, reproduciendo la imagen de "la malvada-perversa-sin escrúpulos" que usa el deseo sexual de ellos para seducirlos y lograr fines concretos, ya que como afirma Asunción Bernárdez,

[...] mujeres y violencia física es un binomio inaceptable porque la feminidad como entidad abstracta -encarnada sin embargo en los cuerpos y los roles sociales- está definida por la imposibilidad de ejercer la violencia física. [...] Las mujeres pueden ser poderosas, e incluso malvadas, pero su poder debe proceder del uso de las "malas artes. (Bernárdez Rodal, 2012, p. 93)

\subsection{Las hubots como objetos sexuales}

Las hubots mujeres vienen programadas con funciones sexuales aunque su cometido sea otro, pero los hubots hombres tienen que ser jaqueados para poder tener funciones sexuales (lo que se considera ilegal). No existe la capacidad de comprar hubots masculinos con funciones sexuales porque en apariencia parece no existir la demanda social de dicho producto para las mujeres. Es central en la serie la red que se dedica a secuestrar hubots parar reprogramarlos y venderlos. Muchas de las hubots robadas y secuestradas son reprogramadas en la serie para la prostitución, en un claro paralelismo con la explotación sexual de mujeres en las sociedades actuales.

Figura 4. Imagen aparecida en la primera temporada de la serie Real Humans (2012), referida a los lugares donde los humanos encuentran sexo con robots programadas como esclavas sexuales

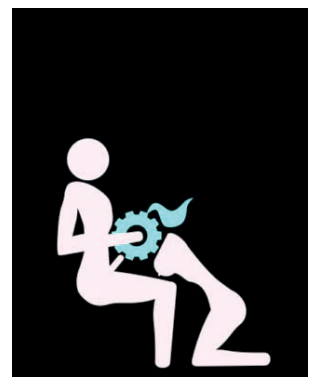




\subsection{Los valores de la sociedad y los valores de la tecnología}

Al final de serie, los hubots consiguen un estatuto jurídico que les equipara en algunos derechos a las personas humanas pero no así en cuestiones como el derecho a ser madres, en donde la esencialización biológica vuelve a imponer su excepcionalidad. La justicia sigue siendo en la serie "la justicia de los hombres" a pesar de que la jueza sea una mujer, porque todo en apariencia es diferente pero reproduce los valores de las sociedades binarias.

Los hubots son en realidad una reproducción de las mentes de quienes los crean, y por lo tanto, de una sociedad que perpetúa la subordinación, la discriminación y la violencia.

\section{Conclusiones}

La tecnología, al fin y al cabo, es obra de mentes humanas que imponen la idolología del poder. Tal y como afirma Audrey Tang, Ministra de lo Digital de Taiwán, el reto será

[...] pedir a la tecnología que construya el sistema de herramientas que refleje los valores que la sociedad desea. Porque si la sociedad no hace este tipo de reflexión, la tecnología tampoco lo hará; se creará un sistema de tecnologías que liberen u opriman a las personas pero sin pensar en sus consecuencias. (Del Castillo, 2016)

Pensar la tecnología con valores implica hacerlo desde una perspectiva de género para centrar la atención, tal y como lo hace Judy Wajcman en su obra Tecnofeminismo (2004), en que los propios artefactos tecnológicos están diseñados por relaciones de género, por sus significados y sus prácticas sociales.

Si el imaginario de "Frankestein" nació en el entorno de la Revolución Industrial (segunda mitad del siglo XVIII), podría decirse que las obras actuales, nacen en consecuencia de una "revolución tecnológica", también denominada "cuarta revolución industrial”.

Los medios de comunicación social son fundamentales en la transmisión de los cambios tecnológicos que se están produciendo. El periodismo, la publicidad, la ficción mediática pueden contribuir a acercar los avances tecnológicos de manera positiva a la ciudadanía, sentando las bases para un debate político, económico y social, desde perspectivas multidisciplinares y con perspectiva de género, sin visiones catastrofistas, sensacionalistas, o de alarma social. La tecnología en sí misma no es ni buena ni mala, depende del uso que se haga de ella. Las máquinas pueden ser utilizadas en la guerra o en la paz, someter o liberar de la opresión.

Cabe resaltar la responsabilidad social de un género como el de la ciencia ficción de marcada crítica social pero que sigue manifestando a través de las películas, los cómic, las novelas, o las series de televisión como la que hemos 
analizado, una actitud conservadora respecto a transgredir las normas y proponer diversos modelos sexo genéricos, identidades, orientaciones, o multiculturalismos. La mayoría de las obras creadas por varones se caracterizan por su inmensurable riqueza de posibilidades y de imaginación empleada, pero en esas mismas obras las mujeres y a las niñas responden más que a modelos plurales de futuro, a un modelo tradicional construido de esencias destacadamente retrógradas y misóginas.

Algunos autores afirman que un país sin ciencia ficción es un país sin futuro, al igual que un país sin ciencia es un país sin futuro o sin esperanza (Unesco), y en esa ciencia y en ese futuro, es prioridad contar con las aportaciones de más de la mitad de la población del mundo que representan las mujeres. Lo que ayer era ciencia ficción hoy es realidad, y lo que hoy imaginamos como ciencia ficción es un futuro cercano y posible. Un futuro diferente y con calidad de vida, dependerá de la construcción de modelos sociales y tecnológicos equitativos. El futuro ya está aquí, y lo exigimos en igualdad.

\section{Referencias bibliográficas}

Amorós, C. (2007). Feminismo y globalización 1987- 2007. En 20 años del Fórum de Política Feminista, XVIII Taller, pp. 13- 21.

Anderson Bonnie S. \& Zinsser J. P. (2009). Historia de las mujeres. Una historia propia. Barcelona: Crítica.

Ansede, M. (2016, octubre 14). Los hombres han ganado el 97\% de los Nobel de ciencia desde 1901. El País. Recuperado de http://bit.ly/2e2ORh2.

Bernárdez Rodal, A. (2012). Modelos de mujeres fálicas del postfeminismo mediático: una aproximación a 'Millenium 1', 'Avatar' y 'Los juegos del hambre'. Anàlisi. Quaderna de comunicaciò (47), pp. 91-112.

Braidotti, R. (2015). Lo Posthumano. Barcelona: Gedisa.

Braidotti, R. (2009). Transposiciones: sobre la ética nómada. Barcelona: Gedisa.

Braidotti, R. (2005). Metamorfosis: hacia una teoría materialista del devenir. Madrid: Akal.

Byrne, M. (2016). El punto de vista futurista del amor de un holograma de 318 años. TED. Recuperado de http://bit.ly/2vMJkjn.

C. Fanjul S. (2015, junio 30). De posthumanos y de hombres. El País. Recuperado de http://bit.ly/2elOgWj.

Cortina A. \& Serra M. Á. (2016, enero 3). Retos de un futuro posthumano. El País. Recuperado de http://bit.ly/1OETkwZ.

Cortina, A. \& Serra, M. Á. (2015). ¿ Humanos o posthumanos? Madrid: Fragmenta.

Del Castillo, C. (2016, diciembre 11). Si la sociedad no reflexiona sobre cuáles son sus valores, la tecnología tampoco lo hará. Público. Recuperado de http://bit. ly/2iPo3jZ. 
EFE (2017, febrero 2). La era en los que los robots serán los mejores aliados del hombre. El Espectador. Recuperado de http://bit.ly/2kemgIX.

EFE Madrid (2016, abril 16). Nuestro futuro dependerá del binomio hombre-máquina. Agencia EFE. Recuperado de http://bit.ly/2eHw3TN.

El sexo con robots está más cerca de lo que crees (2016, diciembre 23). Mundo Deportivo. Recuperado de http://bit.ly/2grKfUB.

Escudero Pérez, J. (2010). Tecnoeroínas: identidades femeninas en la ciencia ficción cinematográfica. Oviedo: KRK Ediciones.

Fernández Romero, D. (2016, mayo 18). Ciberfeminismo, o cómo el cíborg y las chicas disturbio subvierten Matrix. Ctxt. Recuperado de http://bit.ly/2grGnCN.

Gómez, J. A. (2014, julio 22). En 2045 el hombre será inmortal. El Mundo. Recuperado de http://bit.ly/lltKDYL.

Guillén, B. (2016, septiembre, 27). En pocos años no podremos distinguir entre robots y humanos. El País. Recuperado de http://bit.ly/2d4BHxy.

Haraway, D. (1995). Ciencia, cyborg y mujeres. Madrid: Cátedra Feminismos.

Hay estereotipos sexistas: se diagnostica como psicológico lo que es biológico o social (2010, noviembre 22). Diagonal. Recuperado de http://bit.ly/2vM61Ey.

Koval, S. (2008). La condición poshumana. Buenos Aires: Editorial Cinema.

Las prótesis le dan una nueva vida a Jessica (2014, junio 6). El Comercio. Recuperado de http://bit.ly/2wLBkUv.

López Frías, D. (2017, febrero, 26). Así pasé una hora con una muñeca por 80 euros en el prostíbulo de las sex dolls. El Español. Recuperado de http://bit. ly/2lncNfp.

Lorde, A. (2008). Los diarios del cáncer. Rosario: Hipólita Ediciones.

Most, A. (2015, septiembre 16). Meet the bionic model who walked in New York fashion week. Time. Recuperado de http://ti.me/1QieinX.

Mrozfeb J. (2015, febrero, 16). Hand of a superhero. The New York Times. Recuperado de http://nyti.ms/2iLojBn.

Muiña, A. (2016). Mina Loy. Futurismo. Dada. Surrealismo. Madrid: La Linterna Sorda.

Ortega Arjonilla, E.; Rosón, M. \& Platero Méndez, R. (L.) (2017). Barbarismos Queer. Barcelona: Bellaterra.

Pérez Sedeño, E. \& García Dauder, S. (2017). Las mentiras científicas sobre las mujeres. Madrid: La Catarata.

Por más mujeres en tecnología (2016, junio 28). La Nación. Recuperado de http:// bit.ly/2iJEvpP.

¿Podrías llegar a amar a un robot? (2015, 26 diciembre). BBC Mundo. Recuperado de http://bbc.in/1QOk456.

¿Podrías llegar a amar a un robot? (2016, 1 enero). La Nación. Recuperado de http://bit.ly/2glwfbg.

Rius, M. (2016, junio 12). Robofilia: la perspectiva del sexo con robots abre dilemas morales. La Vanguardia. Recuperado de http://bit.ly/2vMigki. 
Rivière, M. (200o, julio 23). La falsa igualdad. El País. Recuperado de http://bit. $\mathrm{ly} / 2 \mathrm{glH} 2 \mathrm{C} 4$.

Rubin, G. (1989). Reflexionando sobre el sexo: notas para una teoría radical de la sexualidad. En Vance, C. S. (ed.). Placer y peligro. Explorando la sexualidad femenina, 113-19o. Madrid: Editorial Revolución. Recuperado de http://bit. ly/2vIkpyd.

Ruiz del Árbol, M. (2016, diciembre 8). La joven perseguida en Afganistán por ayudar a las mujeres con tecnología. El País. Recuperado de http://bit.ly/2hynoY4.

Sampredro, J. (2016, octubre 20). La robotasa. El País. Recuperado de http://bit. ly/2elJNle.

Seoane, A. (2017, marzo 3). Margaret Atwood: Sin la esclavitud Europa sería inviable. El Cultural. Recuperado de http://bit.ly/2wlkria.

Snyder, B. (2016, junio 2). Hugh Herr, el hombre biónico, premio Princesa Asturias de Investigación El País. Recuperado de http://bit.ly/1spMtoo.

Tajahuerce Ángel, I. \& Mateos Casado, C. (2016). Simulaciones sexo genéricas, bebés reborn y muñecas eróticas hiperrealistas. Revista Opción, Año 32, 81, pp. 189-212. Recuperado de http://bit.ly/2estuIf.

Valls- Llobet, C. (2009). Mujer, salud y poder. Madrid: Cátedra Feminismos.

Wajcman, J. (2004). El tecnofeminismo. Madrid: Cátedra Feminismos.

Woolf, V. (2012). Una habitación propia. Madrid: Alianza Editorial.

¿Y si los robots se rebelan contra el hombre? Europa quiere un botón para 'apagarlos' en caso de peligro (2017, enero 12). La Nación. Recuperado de http:// bit.ly/2espQy6. 